\title{
Genetic link between Cabeza, a Drosophila homologue of Fused in Sarcoma (FUS), and the EGFR signaling pathway
}

Mai Shimamura, 2, , Akane Kyotani ${ }^{1,2}$, Yumiko Azuma ${ }^{3}$, Hideki Yoshida ${ }^{1,2}$, Thanh Binh Nguyen ${ }^{1,2}$, Ikuko Mizuta ${ }^{3}$, Tomokatsu Yoshida, Toshiki Mizuno ${ }^{3}$, Masanori Nakagawa ${ }^{4}$ Takahiko Tokuda ${ }^{3,5} *$ and Masamitsu Yamaguchi ${ }^{1,2} *$

${ }^{1}$ Department of Applied Biology and ${ }^{2}$ Insect Biomedical Research Center, Kyoto Institute of Technology, Matsugasaki, Sakyo-ku, Kyoto 606-8585, Japan; ${ }^{3}$ Department of Neurology, ${ }^{4}$ North Medical Center and ${ }^{5}$ Department of Molecular Pathobiology of Brain Diseases, Graduate School of Medical Science, Kyoto Prefectural University of Medicine, 465 Kajii-cho, Kamigyo-ku, Kyoto 602-8566, Japan

*Corresponding authors: M. Yamaguchi, Department of Applied Biology, Kyoto Institute of Technology, Matsugasaki, Sakyo-ku, Kyoto 606-8585, Japan; Tel: +81-75-724-7781, Fax: +81-75-724-7799; E-mail: myamaguc@kit.ac.jp and T. Tokuda, Department of Molecular Pathobiology of Brain Diseases (Neurology), Graduate School of Medical Science, Kyoto Prefectural University of Medicine, 465 Kajii-cho, Kamigyo-ku, Kyoto 602-8566, Japan; Tel: +81-75-251-5793, Fax: +81-75-211-8645; E-mail: ttokuda@koto.kpu-m.ac.jp

\#Present address: Environmental Research Laboratory of Public Health, Kankyo Eisei Yakuhin Co. Ltd., 3-6-2, Hikaridai, Seika-cho, Soraku-gun, Kyoto, Kyoto 619-0237, Japan 


\begin{abstract}
Amyotrophic Lateral Sclerosis (ALS) is a fatal neurodegenerative disease that causes progressive muscular weakness. Fused in Sarcoma (FUS) that has been identified in familial ALS is an RNA binding protein that is normally localized in the nucleus. However, its function in vivo is not fully understood. Drosophila has Cabeza (Caz) as a FUS homologue and specific knockdown of $\mathrm{Caz}$ in the eye imaginal disc and pupal retina using a GMR-GAL4 driver was here found to induce an abnormal morphology of the adult compound eyes, a rough eye phenotype. This was partially suppressed by expression of the apoptosis inhibitor P35. Knockdown of Caz exerted no apparent effect on differentiation of photoreceptor cells. However, immunostaining with an antibody to Cut that marks cone cells revealed fusion of these and ommatidia of pupal retinae. These results indicate that $\mathrm{Caz}$ knockdown induces apoptosis and also inhibits differentiation of cone cells, resulting in abnormal eye morphology in adults. Mutation in EGFR pathway-related genes, such as rhomboid-1, rhomboid-3 and mirror suppressed the rough eye phenotype induced by Caz knockdown. Moreover, the rhomboid-1 mutation rescued the fusion of cone cells and ommatidia observed in Caz knockdown flies. The results suggest that Caz negatively regulates the EGFR signaling pathway required for determination of cone cell fate in Drosophila.
\end{abstract}

Key words: Amyotrophic Lateral Sclerosis, Cabeza, retina, EGFR pathway, Drosophila

Abbreviations: Caz, Cabeza; ALS, Amyotrophic Lateral Sclerosis; FUS, Fused in 
Sarcoma; EGFR, Epidermal growth factor-receptor; SOD1, Cu/Zn superoxide dismutase; TDP-43, TAR DNA-binding protein of 43kDa gene; CNS, central nervous system; APF, after pupal formation; ERK, Extracellular signal-related kinase. 


\section{Introduction}

Amyotrophic lateral sclerosis (ALS) is a fatal neurodegenerative disease that is characterized by degeneration of upper and lower motor neurons of the brain and the spinal cord, which leads to progressive muscle weakness and fatal paralysis [1]. Most cases of ALS are sporadic, but some patients have a familial history as a result of a mutation in the gene for $\mathrm{Cu} / \mathrm{Zn}$ superoxide dismutase (SOD1) [2].

The family of MAPKs includes ERK, p38 and JNK. Each MAPK signaling pathway consists of at least three components, a MAPK kinase kinase, a MAPK kinase and a MAPK. Deviation from strict control of MAPK signaling pathways has been implicated in the development of human neurodegenerative diseases including Alzheimer's, Parkinson's and ALS [3]. Recently it was reported that aberrant expression and activation of $\mathrm{p} 38$ in motor neurons and microglia play important roles in ALS progression [4]. Persistent activation of p38 correlates with degeneration of motor neurons in transgenic mice expressing a mutant SOD1 [5,6]. Moreover a p38 inhibitor was demonstrated to prevent the apoptosis of motor neurons induced by a mutant SOD1 [7]. Thus a possible link between MAPK signaling and ALS has been suggested.

A substantial number of proteins linked to ALS are directly or indirectly involved in RNA processing [8]. Among RNA-binding proteins, mutations in the TAR DNA-binding protein of $43 \mathrm{kDa}$ gene (TDP-43) and fused in sarcoma (FUS) gene have been identified as major genetic causes in both familial and sporadic ALS [9-18]. TDP-43 and FUS are implicated in multiple aspects of RNA metabolism including transcriptional regulation, mRNA splicing and mRNA shuttling between the nucleus and the cytoplasm $[19,20]$.

Drosophila has a single orthologue of human FUS, named Cabeza (Caz). In situ 
hybridization and immunohistochemistrical analyses demonstrated that Caz mRNA and protein are enriched in the brain and central nervous system (CNS) during embryogenesis, and the Caz protein has been detected in the nuclei of several larval tissues and in imaginal discs [21,22]. The full-length recombinant Caz protein and its RRM domain are capable of binding RNA in vitro [21]. These findings suggest that Caz is a nuclear RNA binding protein that may play an important role in the regulation of RNA metabolism during Drosophila development.

In our previous studies using neuron specific Caz knockdown flies, we demonstrated that Caz functions in neuronal cell bodies and/or axons of the CNS and is involved in elongation of synaptic branches of motoneurons [22]. However, contributions of Caz during development of various tissues in Drosophila are not fully understood. As a first step toward clarification, we investigated the effect of knockdown of $\mathrm{Caz}$ on eye development and revealed a rough eye phenotype, accompanied by apoptosis, abnormal differentiation of cone cells and defects in ommatidia rotation. In addition, a Rhomboid-1 mutant could be shown to rescue the fusion of cone cells and mutations of rhomboid-3 and mirror significantly suppressed the rough eye phenotype of the Caz knockdown flies. Since rhomboid-1, rhomboid-3, and mirror are EGFR pathway-related genes, these results indicate genetic links between Caz and EGFR signaling.

\section{Materials and Methods}

\section{Fly stocks}

Fly stocks were maintained at $25^{\circ} \mathrm{C}$ on standard food containing $0.7 \%$ agar, $5 \%$ glucose and $7 \%$ dry yeast. Canton $\mathrm{S}$ was used as the wild type. $w$; UAS-Caz-IR $363-399 ;+$ 
(CG3606) and UAS-rho-IR $R^{28690}$ was obtained from Vienna Drosophila RNAi Center (VDRC). The RNAi of this strain was targeted to the region corresponding to residues 363-399 of Drosophila Caz (UAS-Caz-IR $363-399)$. Four and seven transgenic strains carrying UAS-Caz-IR $1-167$ and $U A S-C a z-I R_{180-346}$ were established, respectively [22]. Each transgenic strain showed a consistent phenotype. Alleles of the following genes were obtained from the Bloomington Drosophila stock center: mirror ${ }^{\text {SaiD3 }}, r u^{1}, r h o^{7 M 43}$ and $r h o^{A A 69}$. Enhancer trap lines carrying the lacZ markers AE127 (inserted into seven-up) [23] and P82 (inserted into deadpan) [24] were obtained from Y. Hiromi. These lines express the $\beta$-galactosidase marker in photoreceptor cells $(\mathrm{R})$ of R3/R4/R1/R6 and R3/R4/R7, respectively. hspFlp; +; tub1> FRT cd2 FRT> GAL4, $U A S-G F P / T M 3$ was a kind gift from A. Plessis. Establishment of lines carrying GMR-GAL4 was as described earlier [25]. Act5C-GAL4/ TM6B was also obtained from the Bloomington Drosophila stock center.

\section{Generation of RNAi clones in retinae}

RNAi clones in retinae were generated with the flip-out system [26]. Female flies with hspFlp; +; tubl> FRT cd2 FRT> GAL4, UAS-GFP/ TM3 were crossed with w; UAS-Caz-IR $363-399 ;+$ male flies and clones were marked by the presence of GFP. Flip-out was induced $24-48 \mathrm{~h}$ after egg laying with a 60 min heat shock at $37^{\circ} \mathrm{C}$.

\section{Immunostaining}

For immunohistochemistory, larval eye imaginal discs and pupal retinae were dissected, and fixed in $4 \%$ paraformaldehyde/ PBS for $15 \mathrm{~min}$ and $30 \mathrm{~min}$ at $25^{\circ} \mathrm{C}$, respectively. After washing with PBS containing 0.3\% Triton X-100, the samples were blocked with PBS containing $0.15 \%$ Triton X-100 and $10 \%$ normal goat serum for 30 min at $25^{\circ} \mathrm{C}$, and incubated with diluted primary antibodies in PBS containing 0.15\% Triton X-100 
and $10 \%$ normal goat serum for $16 \mathrm{~h}$ at $4{ }^{\circ} \mathrm{C}$. The following antibodies were used; mouse anti-LacZ (1:500, Developmental Studies Hybridoma Bank [DSHB], 40-1a), mouse anti-Elav (1:200 DSHB 9F8A9), mouse anti-Cut (1:500, DSHB 2B10), mouse anti-Discs large (1:500) (DSHB) and anti-di-phospho ERK (dpERK) (1: 500) (Sigma). After extensive washing with PBS containing 0.3\% Triton X-100, samples were incubated with secondary antibodies labeled with either Alexa 546 or Alexa 488 (1:400, Invitrogen) for $3 \mathrm{~h}$ at $25^{\circ} \mathrm{C}$. Alexa 488 -conjugated phalloidin (200 units/ ml) was used for the detection of F-actin. After extensive washing with PBS containing $0.3 \%$ Triton X-100, samples were mounted in Vectashield (Vector Laboratories Inc.) and analyzed by confocal laser scanning microscopy (Olympus Flouview FV10i).

\section{Western immunoblot analysis}

Protein extracts from the whole pupae of Drosophila carrying Act5C-GAL4/+ or Act5C-GAL4/+; UAS-Caz-IR $363-399 /+$ were prepared as previously described [22]. The homogenates were boiled at $100{ }^{\circ} \mathrm{C}$ for $5 \mathrm{~min}$, and then centrifuged. The supernatants (extracts) were electrophoretically separated on SDS-polyacrylamide gels containing $12 \%$ acrylamide and then transferred to polyvinylidene difluoride (PVDF) membranes (Bio-Rad). The blotted membranes were blocked with TBS/0.05\% Tween containing $5 \%$ skim milk for $1 \mathrm{~h}$ at $25{ }^{\circ} \mathrm{C}$, followed by incubation with rabbit polyclonal anti-Caz at a 1:5,000 dilution for $16 \mathrm{~h}$ at $4{ }^{\circ} \mathrm{C}$. After washing, the membranes were incubated with HRP-conjugated anti-rabbit IgG (GE Healthcare Bioscience) at 1:10,000 dilution for $2 \mathrm{~h}$ at $25{ }^{\circ} \mathrm{C}$. Antibody binding was detected using ECL Western blotting detection reagents (GE Healthcare Bioscience) and images were analyzed using a Lumivision Pro HSII image analyzer (Aisin Seiki).

\section{Apoptosis assay}


Third instar larvae or 42APF pupae were dissected in PBS and the eye imaginal discs or pupal retinae were fixed in $4 \%$ paraformaldehyde in PBS for $30 \mathrm{~min}$ at $25^{\circ} \mathrm{C}$. After being washed with $0.3 \%$ PBST, the sample were permeabilized by incubation in $0.25 \%$ PBST for $20 \mathrm{~min}$ in $25^{\circ} \mathrm{C}$. After washing with $\mathrm{H}_{2} \mathrm{O}$, the TUNEL reaction was carried out using a Click-iT TUNEL Alexa Fluor 594 Imaging Assay Kit (Life Technologies) according to the manufacture's recommendations.

\section{Scanning electron microscopy}

Adult flies were anesthetized, mounted on stages, and observed under a scanning electron microscope (SEM) VE-7800 (Keyence Inc.) in the low vacuum mode. The eye phenotype of at least five adult male flies ( 3 to 5 days old) of each line was examined in each experiment and the experiments were done in triplicate. No significant variation in eye phenotype was observed among the five individuals.

\section{Data analysis}

Quantification of intensity of Caz signals was carried out with six to nine different samples by using Meta Morph software (Molecular Devices). For the statistical analysis, Microsoft Excel 2007 was used. P-values were calculated using Welch's t-test and the error bars represent Standard Errors from Means.

\section{Results}

\section{Knockdown of $\mathrm{Caz}$ in eye imaginal discs induces morphologically aberrant rough} eyes

In order to investigate in vivo functions of Caz, we examined the effect of reduction of 
Caz protein in vivo using a combination of the GAL4-UAS targeted expression system and the RNAi method. Knockdown of Caz in all tissues by the Act5C-GAL4 driver strain resulted in late pupal lethality in transgenic lines carrying $U A S-C a z-I R_{1-167}$ (data not shown). Knockdown of $\mathrm{Caz}$ in eye imaginal discs by the GMR-GAL4 driver strain, in which Caz double-stranded RNA (dsRNA) was expressed in the region posterior to the morphogenetic furrow, induced morphologically aberrant rough eyes. SEM images showed fusion of ommatidia and a lack of bristles (Fig. S1C-F). Flies carrying GMR-GAL4 alone exhibited apparently normal eye morphology (Fig. S1A, B). Moreover, to eliminate the possibility of off-target effects, we established eleven independent transgenic fly lines carrying $U A S$-Caz-IR targeted to the different regions of the Caz mRNA assessed in a previous study [22]. Phenotypes of the established transgenic fly lines crossed with the GMR-GAL4 driver strain are reported [27]. Each independent strain showed the similar rough eye phenotype as the flies carrying UAS-Caz-IR targeted to different regions of the Caz mRNA (Fig. S1C-F). These results suggest that the rough eye phonotype observed in $\mathrm{Caz}$ knockdown flies is not due to a possible insertional mutation or off-target effect but rather to reduction of the Caz protein level. Throughout the following studies, we utilized the strain CG3606 carrying UAS-Caz-IR 363-399.

To investigate whether the expression of $\mathrm{Caz}$ dsRNA efficiently reduces the level of Caz protein, we performed immunostaining of pupal retinae at $42 \mathrm{~h}$ after pupal formation (APF) with anti-Caz antibodies. We utilized the flip-out system to produce the RNAi clone so that the level of Caz could be directly compared within a single retina. We used UAS-Caz-IR $363-399$ line, since it apparently showed the severest rough eye (Fig. S1E and F). Within the RNAi clone of Caz marked by the presence of GFP 
signals, Caz signals marked by Red were reduced by $50 \%$ (Fig. 1A to G). Double immunostaining of pupal retinae with anti-cut antibody and ant-Caz antibody revealed relatively high expression of Caz in cone cells (Fig. 1J) and the Caz signal was reduced by $62 \%$ in flies expressing Caz dsRNA drived by GMR-GAL4 (Fig. 1K and N). Moreover, effective knockdown of $\mathrm{Caz}$ in pupae of $U A S$-Caz-IR $363-399$ line was demonstrated by the Western immunoblot analysis with anti-Caz antibody (Fig. 1O). All of these results further indicate that the rough eye phenotype observed in RNAi flies of Caz is due to reduction of the Caz protein level.

\section{Knockdown of $\mathrm{Caz}$ induces apoptosis in pupal retinae}

Extensive apoptosis could be considered as one factor causing fused ommatidia in the adult compound eye, since it is frequently accompanies this phenotype. We therefore examined if excessive cell death might occur during eye development in $\mathrm{Caz}$ knockdown flies by crossing GMR-GAL4; UAS-Caz-IR $363-399$; + with flies expressing a broad specificity Caspase inhibitor P35 encoded by the baculovirus Autographa californica [28]. Partial suppression of the rough eye phenotype induced by knockdown of Caz was observed in flies co-expressing P35 (Fig. 2A, a-f). Expression of P35 alone exerted no apparent effect on the compound eye morphology (Fig. 2A, g and h). Moreover, we monitored apoptotic cells in third instar larval eye imaginal discs by TUNEL assay. However, no detectable apoptotic signals in eye imaginal discs of these flies was observed (Fig. 2B, a, d, g and j). We therefore next examined pupal retinae at $42 \mathrm{~h}$ APF by TUNEL assay. Apoptotic cells detected in the Caz knockdown retinae were significantly reduced in flies expressing P35 (Fig. 2B, c, f and i). These results indicate that knockdown of $\mathrm{Caz}$ induces apoptosis in some cells in pupal retinae. 


\section{Knockdown of $\mathrm{Caz}$ interferes with cell differentiation in pupal retinae}

Photoreceptor cells are known to be generated in a stereotype order: R8 is generated first, with movement posterior from the morphogenetic furrow, then cells are added pair wise (R2 and R5, R3 and R4, and R1 and R6), and R7 is the last photoreceptor to be added to each cluster [29]. To investigate whether Caz knockdown inhibits differentiation of photoreceptor cells, we crossed flies expressing Caz dsRNA with two enhancer trap lines, AE127 and P82, to specifically mark photoreceptor cells of $\mathrm{R} 3 / \mathrm{R} 4 / \mathrm{R} 1 / \mathrm{R} 6$ and R3/R4/R7, respectively and then immunostained the eye imaginal discs with anti- $\beta$-galactosidase antibodies (Fig. S2, A-B and D-E). In parallel, we carried out immunostaining of eye imaginal discs with anti-Elav antibodies (Fig. S2, C, F). Elav, a pan-neuronal marker is normally expressed in the posterior portion of the eye imaginal discs. In eye imaginal discs of Caz knockdown flies, all eight photoreceptor cells and their neuron appeared to differentiate normally (Fig. S2).

We next examined the pattern formation in pupal ommatidia of Caz knockdown flies. Differentiation of photoreceptors, cone cells, and $1^{\circ}, 2^{\circ}$, and $3^{\circ}$ pigment cells was completed by about $42 \mathrm{~h}$ APF at $28^{\circ} \mathrm{C}$. However, in pupal retinae of $\mathrm{Caz}$ knockdown flies, immunostaining with an anti-Cut antibody that marks cone cells showed that some of these were fused in flies expressing Caz dsRNA (Fig. 3D and H). Flies carrying GMR-GAL4 alone exhibited apparently normal cone cells (Fig. 3A and H). The quantified data indicate that occurrence of cone cell fusion was increased by 5.7 fold in the $C a z$ knockdown retinae.

Furthermore, we monitored apical cell junctions in pupal retina at $42 \mathrm{~h}$ APF by immunostaining with anti-Discs large. The results showed cells in pupal retinae of $\mathrm{Caz}$ 
knockdown flies to be attached loosely, the orientation of ommatidia to be irregular, and the size of ommatidia to vary (Fig. 4C). In addition some ommatidia were apparently fused (Fig. 4C). These data suggest that knockdown of $\mathrm{Caz}$ disrupts differentiation of pupal ommatidial cell types, especially cone cells, and $1^{\circ}, 2^{\circ}$, and $3^{\circ}$ pigment cells, probably by repressing or enhancing expression of genes involved in differentiation processes.

\section{Genetic link between $\mathrm{Caz}$ and rhomboid}

The epidermal growth factor-receptor (EGFR) signaling pathway, evolutionarily conserved from $C$. elegans to man, controls a variety of different cellular processes. In Drosophila, these include proliferation, patterning, cell-fate determination, migration, and survival [30]. Contributions to cone cell-fate and ommatidial rotation have also been documented [30-33]. One of the rate limiting components of Drosophila EGFR signaling is Rhomboid [32-34]. We therefore examined the effects of mutations that might modify the Caz-induced rough eye phenotype, especially focusing on the rhomboid gene.

Half dose reduction rhomboid-1 (rho) significantly suppressed the rough eye phenotype (Fig. 5A, a, b, c and d) and rescued the fusion of cone cells (Fig. 3E and H) in pupal retinae. Rescue of the fusion of the cone cells was also observed with overexpression of P35 (Fig. 3G and $\mathrm{H}$ ), suggesting that induction of apoptosis is also responsible for this phenotype. Two different alleles of rho showed suppression of the rough eye (Fig. 5A, a, b, c and d). In addition, similar extent of suppression of the rough eye was observed by knockdown of rho (Fig. 5A, $m$ and $n$ ), but not by expression of dsRNA for GFP (Fig. 5A, k and 1). Rhomboid-1 is a seven membrane-spanning serine 
protease, undergoing cleavage of Spitz to release the secreted form as an EGFR ligand from the Golgi apparatus [32-35]. In the eye, Rhomboid-3, also known as Roughoid, cooperates with Rhomboid-1 [32, 33]. Expectedly, the Rhomboid-3 hypomorph mutant $r u^{l}$ also demonstrated suppression of the rough eye phenotype induced by knockdown of $\mathrm{Caz}$ (Fig. 5A, g and h). Moreover, a mirror loss of function mutant mirr ${ }^{\text {SaiD3 }}$ similarly suppressed the rough eye phenotype (Fig. 5A, e and f). The mirror gene encodes a homeodomain-containing transcription factor that is thought to activate transcription of rhomboid [36]. In addition, immunostaining of eye imaginal discs with anti-dpERK antibody also pointed activation of ERK signals in the Caz knockdown flies (Fig. 5B). These results indicate a genetic link between Caz and the EGFR signaling pathway.

\section{Discussion}

In this study, we found that Caz knockdown in eye imaginal discs induces a rough eye phenotype associated with apoptosis, abnormal differentiation of cone cells and pigment cells, and defects in ommatidia rotation in pupal retinae. However, apoptosis and differentiation of photoreceptor cells were not affected in larval eye imaginal discs expressing $\mathrm{Caz}$ dsRNA. Why did Caz knockdown in eye imaginal discs affect pupal retinae but not third instar larval eye discs? In situ hybridization and immunohistochemistrical analyses demonstrated that Caz mRNA and protein are enriched in the brain and CNS during embryogenesis, and Caz protein was detected in the nuclei of several larval tissues and in imaginal discs [21]. However, the expression level of Caz is higher in adult eyes than in larval eye discs (Flybase). Thus, it is possible that Caz plays a more important role in eye development in the pupal stage. 
The observation that the rough eye phenotype of Caz knockdown flies was significantly suppressed by co-expression of P35 and that apoptotic cells detected by immunostaining with anti-cleaved Caspase-3 antibody were significantly increased in pupal retinae of flies expressing $C a z$ dsRNA suggests that induction of apoptosis at least partially contributes to the rough eye phenotype. It is reported that the number of dying cells increases dramatically if interactions between cells are disrupted, for instance upon cell ablation [37]. Therefore, one possible explanation is that Caz knockdown disrupts interactions between cells in pupal retinae, as evidenced with anti-Cut immunostaining, that results in induction of apoptosis. In addition, it is well known that apoptosis is induced by JNK or p38 signaling [38-40]. It is also reported that persistent activation of the JNK or p38 signaling pathways mediates neuronal apoptosis in ALS [3-7,41], and that TDP-43 is related to JNK signaling [42]. Thus, another possible explanation is that Caz knockdown induces JNK or p38 signaling, resulting in increase of apoptosis in pupal retinae.

We found a genetic interaction between Caz and Rhomboid, a rate-limiting component of the EGFR signaling pathway. Appropriate levels of EGFR signaling are required for cone cell-fate and ommatidial rotation [30-33]. Knockdown of Caz in eye imaginal discs and pupal retinae induced abnormal differentiation of cone cells and defects in ommatidia rotation that eventually resulted in the rough eye phenotype in adults. The rhomboid-1 mutant rescued the fusion of cone cells and mutations of rhomboid-3 and mirror significantly suppressed the rough eye phenotype of Caz knockdown flies. In contrast, mutations of egfr did not suppress the rough eye phenotype induced by knockdown of Caz (data not shown). These apparently contradictory results might be explained as follows. Once activated, the signaling 
cascade could be amplified progressively, so that only a half reduction of some components of pathway such as egfr may not be sufficient to suppress the effects of over-activation of the initiator such as rhomboid. In any event, the present study suggests that Caz negatively regulates EGFR signaling. Since the expression level of $\mathrm{Caz}$ is much higher in adult eyes than larval eye discs, negative regulation of EGFR signaling by Caz may play a role in controlling EGFR signaling less reactive to oxidative stress during adulthood. It should be noted that a hallmark of ALS is chronic neuronal exposure to oxidative stress and inflammation.

In summary, we have shown that knockdown of Caz in the Drosophila retina induces a rough eye phenotype associated with increased apoptosis, abnormal differentiation of cone cells and pigment cells, and defects in ommatidia rotation. Here we provide the first definitive evidence that Caz plays an important role in regulation of the EGFR signaling pathway. It should be noted that the neurodegeneration occurring in ALS can be accounted for deviation from strict control of MAPK signaling [3]. Thus, the $\mathrm{Caz}$ knockdown flies used in the present study should provide a useful tool for elucidating functions of FUS and pathological mechanisms of associated ALS.

\section{Acknowledgements}

We thank Dr. Y. Hiromi and Dr. A. Plessis for supplying fly lines, and Dr. M. Moore for comments on the English in the manuscript. This study was partially supported by a scholarship and grants from JST and the Ministry of Education, Science, Sports and Culture of Japan and the JSPS Core-to-Core Program, B. Asia-Africa Science Platforms. 


\section{References}

[1] S. Boillee, C. Vande Velde, D.W. Cleveland, ALS: a disease of motorneurons and their nonneuronal neighbors. Neuron 52 (2006) 39-59.

[2] S. Vucic, M.C. Kiernan, Pathology of neurodegeneration in familial amyotrophic lateral sclerosis. Curr Mol Med. 9 (2009) 255-272.

[3] E.K. Kim, E.-J. Choi, Pathological roles of MAPK signaling pathways in human diseases. Biochim. Biophys. Acta 1802 (2010) 396-405.

[4] C. Bendotti, M. Bao Cutrona, C. Cheroni, G. Grignaschi, D. Lo Coco, M. Peviani, M. Tortarolo, P. Veglianese, E. Zennaro, Inter- and intracellular signaling in amyotrophic lateral sclerosis: role of p38 mitogen-activated protein kinase. Neurodegener. Dis. 2 (2005) 128-134.

[5] M. Tortarolo, P. Veglianese, N. Calvaresi, A. Botturi, C. Rossi, A. Giorgini, A. Migheli, C. Bendotti, Persistent activation of p38 mitogen-activated protein kinase in a mouse model of familial amyotrophic lateral sclerosis correlates with disease progression, Mol. Cell. Neurosci. 23 (2003) 180-192.

[6] S.S. Holasek, T.M. Wengenack, K.K. Kandimalla, C. Montano, D.M. Gregor, G.L. Curran, J.F. Poduslo, Activation of the stress-activated MAP kinase, p38, but not JNK in cortical motor neurons during early presymptomatic stages of amyotrophic lateral sclerosis in transgenic mice, Brain Res. 1045 (2005) 185-198.

[7] M. Dewil, V.F. dela Cruz, L. Van Den Bosch, W. Robberecht, Inhibition of p38 mitogen activated protein kinase activation and mutant SOD1(G93A)-induced motor neuron death, Neurobiol. Dis. 26 (2007) 332-341.

[8] R. Lemmens, M.J. Moore, A. Al-Chalabi, R.H. Brown Jr., W. Robberecht, RNA metabplism and the pathogenesis of motor neuron diseases, Trends Neurosci. 33 (2010) 249-258. 
[9] I.R. Mackenzie, R. Rademakers, M. Neumann, TDP-43 and FUS in amyotrophic lateral sclerosis and frontotemporal dementia, Lancet. Neurol. 9 (2010) 995-1007.

[10] M.A. Gitcho, R.H. Baloh, S. Chakraverty, K. Mayo, J.B. Norton, D. Levitch, K.J. Hatanpaa, C.L. White, 3rd., E.H. Bigio, R. Caselli, et al. TDP-43 A315T mutation in familial motor neuron disease, Ann. Neurol. 63 (2008) 535-538.

[11] A. Yokoseki, A. Shiga, C.F. Tan, A. Tagawa, H. Kaneko, A. Koyama, H. Eguchi, A. Tsujino, T. Ikeuchi, A. Kakita, et al., TDP-43 mutation in familial amyotrophic lateral sclerosis, Ann. Neurol. 63 (2008) 538-542.

[12] E. Kabashi, P.N. Valdmanis, P. Dion, D. Spiegelman, B.J. McConkey, C. Vande, Velde, J.P. Bouchard, L. Lacomblez, K. Pochigaeva, F. Salachas, et al., TARDBP mutations in individuals with sporadic and familial amyotrophic lateralsclerosis, Nat. Genet. 40 (2008) 572-574.

[13] J. Sreedharan, I.P. Blair, V.B. Tripathi, X. Hu, C. Vance, B. Rogelj, S. Ackerley, J.C. Durnall, K.L. Williams, E. Buratti, et al., TDP-43 mutations in familialand sporadic amyotrophic lateral sclerosis, Science 319 (2008) 1668-1672.

[14] G.S. Pesiridis, V.M. Lee, J.Q. Trojanowski, Mutations in TDP-43 link glycine-rich domain functions to amyotrophic lateral sclerosis. Hum. Mol. Genet. 18 (2009) R156-R162.

[15] T.J. Kwiatkowski Jr., D.A. Bosco, A.L. Leclerc, E. Tamrazian, C.R. Vanderburg, C. Russ, A. Davis, J. Gilchrist, E.J. Kasarskis, T. Munsat, et al., Mutations in the FUS/TLS gene on chromosome 16 cause familial amyotrophic lateral sclerosis, Science 323 (2009) 1205-1208.

[16] C. Vance, B. Rogelj, T. Hortobagyi, K.J. De Vos, A.L. Nishimura, J. Sreedharan, X. Hu, B. Smith, D. Ruddy, P. Wright, et al., Mutations in FUS, an RNA processing 
protein, cause familial amyotrophic lateral sclerosis type 6, Science 323 (2009) $1208-1211$

[17] C. Hewitt, J. Kirby, J.R. Highley, J.A. Hartley, R. Hibberd, H.C. Hollinger, T.L. Williams, P.G. Ince, C.J. McDermott, P.J. Shaw, Novel FUS/TLS mutations and pathology in familial and sporadic amyotrophic lateral sclerosis, Arch. Neurol. 67 (2010) 455-461.

[18] R. Rademakers, H. Stewart, M. Dejesus-Hernandez, C. Krieger, N. Graff-Radford, M. Fabros, H. Briemberg, N. Cashman, A. Eisen, I.R. Mackenzie, Fus gene mutations in familial and sporadic amyotrophic lateral sclerosis, Muscle Nerve, 42 (2010) 170-176.

[19] H. Zinszner, J. Sok, D. Immanuel, Y. Yin, D. Ron, TLS (FUS) binds RNA in vivo and engages in nucleo-cytoplasmic shuttling, J. Cell Sci., 110 (1997) 1741-1750.

[20] C. Lagier-Tourenne, M. Polymenidou, D.W. Cleveland, TDP-43 and FUS/TLS: emerging roles in RNA processing and neurodegeneration, Hum. Mol. Genet. 19 (2010) R46-R64.

[21] D.T. Stolow, S.R. Haynes, Cabeza, a Drosophila gene encoding a novel RNA binding protein, shares homology with EWS and TLS, two genes invoved in human sarcoma formation, Nucleic Acids Res. 23 (1995) 835-843.

[22] H. Sasayama, M. Shimamura, T. Tokuda, Y. Azuma, T. Yoshida, T. Mizuno, M. Nakagawa, N. Fujikake, Y. Nagai, M. Ymaguchi, Knockdown of the Drosophila Fused in Sarcoma (FUS) homologue causes deficient locomotive behavior and shortening of motoneuron terminal branches, PLoS One, 7 (2012) e39483.

[23] M. Mlodzik, Y. Hiromi, U. Weber, C.S. Goodman, G.M. Rubin, The Drosophila seven-up gene, a member of the steroid receptor gene superfamily, controls 
photoreceptor cell fates, Cell 60 (1990) 211-224.

[24] S. Kramer, S.R. West, Y. Hiromi, Cell fate control in the Drosophila retina by the orphan receptor seven-up: its role in the decisions mediated by the ras signaling pathway, Development 121 (1995) 1361-1372.

[25] Y. Takahashi, F. Hirose, A. Matsukage, M. Yamaguchi, Identification of three conserved regions in the DREF transcription factors from Drosophila melanogaster and Drosophila virilis, Nucleic Acids Res. 27 (1999) 510-516.

[26] J. Sun, J. Tower, FLP recombinase-mediated induction of $\mathrm{Cu} / \mathrm{Zn}$ superoxide dismutase transgene expression can extend the life span of adult Drosophila melanogaster, Mol. Cell. Biol. 19 (1999) 216-228.

[27] Y. Azuma, T. Tokuda, M. Shimamura, A. Kyotani, H. Sasayama, T. Yoshida, I. Mizuta, T. Mizuno, M. Nakagawa, N. Fujikake, M. Ueyama, Y. Nagai, M. Yamaguchi, Identification of ter94, Drosophila VCP, as a strong modulator of motor neuron degeneration induced by knockdown of Caz, Drosophila FUS, Hum. Mol. Genetics (2014) in press.

[28] N.E. Crook, R.J. Clem, L.K. Miller, An apoptosis-inhibiting baculovirus gene with a zinc finger-like motif. J. Virol. 67(1993) 2168-2174.

[29] S.L. Zipursky, G.M. Rubin, Determination of neuronal cell fate: lessons from the R7 neuron of Drosophila, Annu. Rev. Neurosci. 17 (1994) 373-397.

[30] K. Gaengel, M. Mlodzik, Egfr signaling regulates ommatidial rotation and cell motility in the Drosophila eye via MAPK/Pnt signaling and the Ras effector Canoe/AF6, Development 130 (2003) 5413-5423.

[31] W. Fu, M. Noll, The Pax2 homolog sparkling is required for development of cone and pigment cells in the Drosophila eye, Genes Dev. 11 (1997) 2066-2078. 
[32] J.D.Wasserman, S. Urban, M. Freeman, A family of rhomboid-like genes: Drosophila rhomboid-1 and roughoid/rhomboid-3 cooperate to activate EGF receptor signaling, Genes. Dev., 14 (2000) 1651-1663.

[33] B.Z. Shilio, Regulating the dynamics of EGF receptor signaling in space and time. Development, 132 (2005) 4017-4027.

[34] S. Yogev, E.D. Schejter, B.Z. Shilo, Drosophila EGFR signaling is modulated by differential compartmentalization of Rhomboid intramembrane proteases. EMBO J. 27 (2008) 1219-1230.

[35] S. Urban, J. R. Lee, M. Freeman, Drosophila rhomboid-1 defines a family of putative intramembrane serine proteases, Cell 107 (2001) 173-182.

[36] K.C. Jordan, N.J. Clegg, J.A. Blasi, A.M. Morimoto, J. Sen, D. Stein, H. McNeill, W.M. Deng, M. Tworoger, H. Ruohola-Baker, The homeobox gene mirror links EGF signalling to embryonic dorso-ventral axis formation through notch activation, Nat. Genet. 24 (2000) 429-433.

[37] A. Hidalgoa, C.F. Constant, The control of cell number during central nervous system development in flies and mice, Mech. Dev., 120 (2003) 1311-1325.

[38] F. Agnè, M. Suzanne, S. Noselli, The Drosophila JNK pathway controls the morphogenesis of imaginal discs during metamorphosis, Development 126 (1999) 5453-5462.

[39] M. Tare, R.M. Modi, J.J. Nainaparampil, O.R. Puli, S. Bedi, P. Fernandez-Funez, M. Kango-Singh, A. Singh, Activation of JNK signaling mediates amyloid- $\beta$-dependent cell death, PLoS One 6 (2011) e24361.

[40] S.A.Correa, K.L. Eales, The role of p38 MAPK and its substrates in neuronal plasticity and neurodegenerative disease, J. Signal. Transduct. 2012 (2012) 649079. 
[41] S. Ackerley, A.J. Grierson, S. Banner, M.S. Perkinton, J. Brownlees, H.L. Byers, M. Ward, P. Thornhill, K. Hussain, J.S. Waby, B.H. Anderton, J.D. Cooper, C. Dingwall, P.N. Leigh, C.E. Shaw, C.C. Miller, p38 $\alpha$ stress-activated protein kinase phosphorylates neurofilaments and is associated with neurofilaments pathology in amyotrophic lateral sclerosis. Mol. Cell. Neurosci. 26 (2004) 354-364.

[42] J. Meyerowitz, S.J. Parker, L.J. Vella, D.Ch. Ng, K.A. Price, J.R. Liddell, A. Caragounis, Q.X. Li, C.L. Masters, T. Nonaka, M. Hasegawa, M.A. Bogoyevitch, K.M. Kanninen, P.J. Crouch, A.R.White, C-Jun N-terminal kinase controls TDP-43 accumulation in stress granules induced by oxidative stress, Mol. Neurodegener. 6 (2011) 57. 


\section{Figure legends}

Fig. 1-The level of anti-Caz signals is reduced in Caz dsRNA-expressing areas. Immunostaining of retinae at $42 \mathrm{~h}$ APF with anti-Caz antibody $(\mathrm{C}, \mathrm{D}, \mathrm{J}$ and $\mathrm{K})$. RNAi clones in retinae were generated with the flip-out system (A to F). Female flies with hspFlp; +; tubl> FRT cd2 FRT> GAL4, UAS-GFP were crossed male flies with w; UAS-Caz-IR $363-399 ;+$. The Caz dsRNA-expressing area is positively marked with GFP (A, B). (E and F) Merged images. Panels B, D and F show higher magnification images of the regions marked with squares in panels A, C and D. (G) Quantification of intensities of Caz-signals in GFP-positive and -negative areas. Mean intensities with standard deviation from six pupal retinae are shown. $* \mathrm{P}<0.05$. ( $\mathrm{H}$ to $\mathrm{M})$ Immunostaining of retinae at $42 \mathrm{~h}$ APF with anti-cut (H and I) and anti-Caz (J and K) antibodies. (L and M) Merged images. (H, J and $\mathrm{K}$ ) GMR-GAL4/y. (I, K and M) GMR-GAL4/y; UAS-Caz-IR $363-399$. (N) Quantification of intensities of Caz-signals. Mean intensities with standard deviation from nine pupal retinae are shown. $* \mathrm{P}<0.05$. The bars indicate $100 \mu \mathrm{m}(\mathrm{A}, \mathrm{C}$ and $\mathrm{E}), 10 \mu \mathrm{m}(\mathrm{B}, \mathrm{D}$ and $\mathrm{F}$ ) and $20 \mu \mathrm{m}$ (H to $\mathrm{M})$, respectively. (O) Western immunoblot analysis. Protein extracts were prepared from the whole pupae of Drosophila carrying Act5C-GAL4/+ (left lane) or Act5C-GAL4/+; $U A S-C a z-I R_{363-399 /+}$ (right lane). The blot was probed with anti-Caz antibody.

Fig. 2-Knockdown of $\mathrm{Caz}$ in eye imaginal discs induces apoptosis. (A) Over-expression of P35 suppresses the rough eye phenotype, as shown by scanning electron micrographs of adult compound eyes. (a, b) GMR-GAL4/y; UAS-Caz-IR $363-399 /$ +; +, (c, d) GMR-GAL4/y; UAS-Caz-IR $363-399 /+; U A S-G F P /+, \quad(\mathrm{e}, \mathrm{f})$ GMR-GAL4/y; 
UAS-Caz-IR $363-399 /+; \quad U A S-P 35 /+$. (g, h) GMR-GAL4/y; +/+; UAS-P35/+. The flies were developed at $28^{\circ} \mathrm{C}$. The eye phenotype of at least five adult male flies ( 3 to 5 days old) of each line was examined and the experiments were done in triplicate. No significant variation in eye phenotype was observed among the five individuals. The rough area of the compound eye was circled as an index of the rough eye phenotype. The bars indicate $50 \mu \mathrm{m}(\mathrm{a}, \mathrm{c}$, e and $\mathrm{g})$, and $14.2 \mu \mathrm{m}(\mathrm{b}, \mathrm{d}, \mathrm{f}$ and $\mathrm{h})$, respectively. (B) Detection of apoptotic cells in third larval eye imaginal discs (a, d, g and j) and pupal retinae (c, f, i and l) by TUNEL assay. Pupal retinae were also stained with DAPI (b, e, $\mathrm{h}$ and k). (a to c) GMR-GALA/y; UAS-Caz-IR $363-399 /$ +; +. (d to f) GMR-GAL4/y; $U A S-C a z-I R_{363-399 /++} \quad U A S-G F P /+. \quad$ g to i) GMR-GALA/y; UAS-Caz-IR $363-399 /+$; $U A S-P 35 /+$. (j to 1) GMR-GALA/y; +/+; UAS-P35/+. The flies were developed at $28^{\circ} \mathrm{C}$. The bars indicate $100 \mu \mathrm{m}$.

Fig. 3-Knockdown of $\mathrm{Caz}$ interferes with differentiation of cone cells. Immunostaining of retinae at $42 \mathrm{~h} \mathrm{APF}$ with an antibody to Cut that marks cone cells (green). (A) GMR-GAL4/y. (B) GMR-GALA/y; +; UAS-P35/+. (C) GMR-GALA/y; +; $r h o^{A A 69} /+. \quad$ (D) $\quad G M R-G A L 4 / y ; \quad U A S-C a z-I R_{363-399} /+; \quad+. \quad$ (E) GMR-GALA/y; $U A S-C a z-I R_{363-399} /+; r h o^{A A 69} /+$. (F) GMR-GALA/y; UAS-Caz-IR $363-399 /+; \quad U A S-G F P /+$. (G) GMR-GAL4/y; UAS-Caz-IR363-399/+; UAS-P35/+. The flies were developed at $28^{\circ} \mathrm{C}$. Arrows show fused cone cells and fused ommatidia are circled. The bar indicates $20 \mu \mathrm{m}$ (A-G). (H) Quantification of fused cone cells in pupal retinae. Ratio of fused cone cells in each retina us shown (\%). Mean values with standard deviation from six pupal retinae are shown. ${ }^{*} \mathrm{P}<0.05$. (Left to Right) GMR-GAL4/y, GMR-GAL4/y; +; UAS-P35/+, GMR-GALA/y; +; rho ${ }^{A A 69} /+$, GMR-GALA/y; UAS-Caz-IR $363-399 /+; \quad+$, GMR-GALA/y; 
$U A S-C a z-I R_{363-399 /+;} r h o^{A A 69} /+$, GMR-GALA/y;UAS-Caz-IR $363-399 /+; U A S-G F P /+$ and GMR-GAL4/y; UAS-Caz-IR $363-399 /+; U A S-P 35 /+$.

Fig. 4-Effects of $\mathrm{Caz}$ knockdown on morphogenesis of pupal retinae. Confocal sections stained with anti-Discs large (A, C). (A) GMR-GAL4/y. (C) GMR-GAL4/y; $U A S-C a z-I R_{363-399} /+;+$. Black arrows in the lower panels $(\mathrm{B}, \mathrm{D})$ indicate the orientation of the ommatidia. Note disruption in the Caz knockdown flies (D) in compared to control (B). The size of ommatidia is also irregular, and cell attachment appears to be loose (compare panels $\mathrm{A}$ and $\mathrm{C}$ ). The white arrow in panel $\mathrm{C}$ indicates an example of the fused ommatidia.

Fig. 5-Scanning electron micrographs of adult compound eyes. (A) Female flies expressing Caz dsRNA (GMR-GAL4/GMR-GAL4; UAS-Caz-IR $363-399$; +) were crossed with UAS-GFP-IR, UAS-rho-IR ${ }^{28690}, \quad r h o^{7 M 43}$ (amorph), rho ${ }^{A A 69}$ (undetermined), mirror $^{\text {SaiD3 }}$ (loss of function), or $r u^{l}$ (hypomorph) male flies, and then F1 progeny were developed at $28^{\circ} \mathrm{C}$ without balancer chromosomes and used for inspection of the eye phenotype. The eye phenotype of at least five adult male flies (3 to 5 days old) of each line was examined and the experiments were done in triplicate. No significant variation in eye phenotype was observed among the five individuals. The rough area of the compound eye was circled as an index of the rough eye phenotype. (a, b) GMR-GAL4/y; $U A S-C a z-I R_{363-399} /+; r h o^{7 M 43} /+$. (c, d) GMR-GAL4/y; UAS-Caz-IR $363-399 /+; r h o^{A A 69} /+$. (e, f) GMR-GAL4/y; UAS-Caz-IR $363-399 /$ +; mirror $^{\text {SaiD3 } / ~+~(g, ~ h) ~ G M R-G A L 4 / y ; ~}$ $U A S-C a z-I R_{363-399 /}+; \quad r u^{l} /$ +. (i, j) GMR-GAL4/ y; UAS-Caz-IR $363-399 /+; \quad+. \quad(\mathrm{k}, 1)$ GMR-GALA/y; UAS-Caz-IR $363-399 /+; \quad U A S-G F P-I R /+, \quad(\mathrm{m}, \quad$ n) $\quad$ GMR-GALA/y; 
UAS-Caz-IR $363-399 /+; U A S-r h o-I R^{28690 /+}$. The flies were developed at $28^{\circ} \mathrm{C}$. The bar indicates $50 \mu \mathrm{m}(\mathrm{a}, \mathrm{c}, \mathrm{e}, \mathrm{g}, \mathrm{i}, \mathrm{k}, \mathrm{m})$ or $14.2 \mu \mathrm{m}(\mathrm{b}, \mathrm{d}, \mathrm{f}, \mathrm{h}, \mathrm{j}, \mathrm{l}, \mathrm{n})$. (B) Immunostaining of eye imaginal discs with anti-dpERK antibody. (a) GMR-GALA/y. (b) GMR-GALA/ y; UAS-Caz-IR $363-399 /+; \quad+$. Arrows indicate morphogenetic furrows (MF). The bars indicate $100 \mu \mathrm{m}$. 

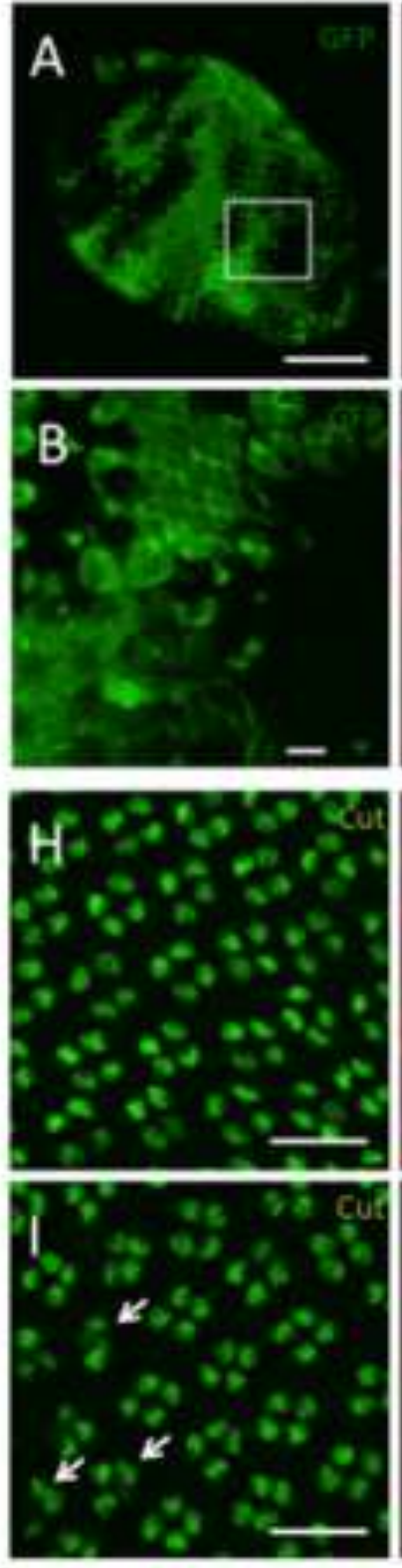
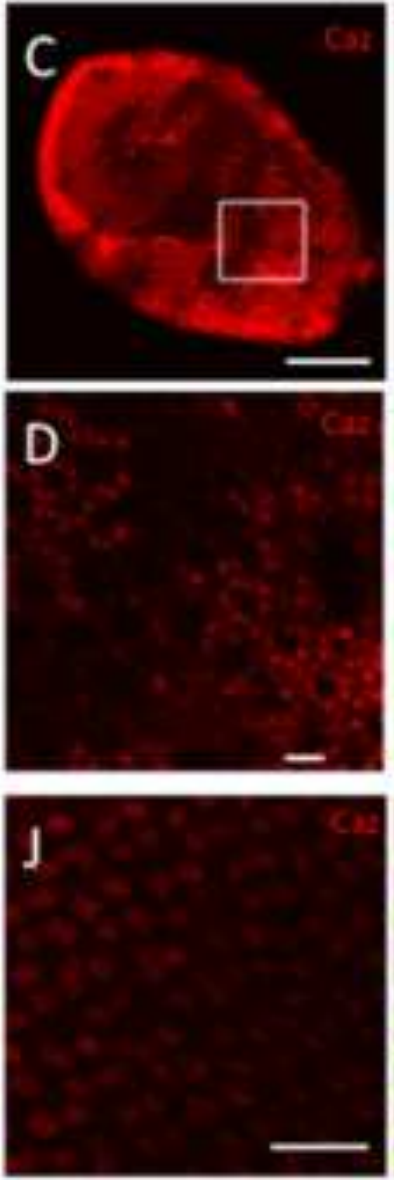

K
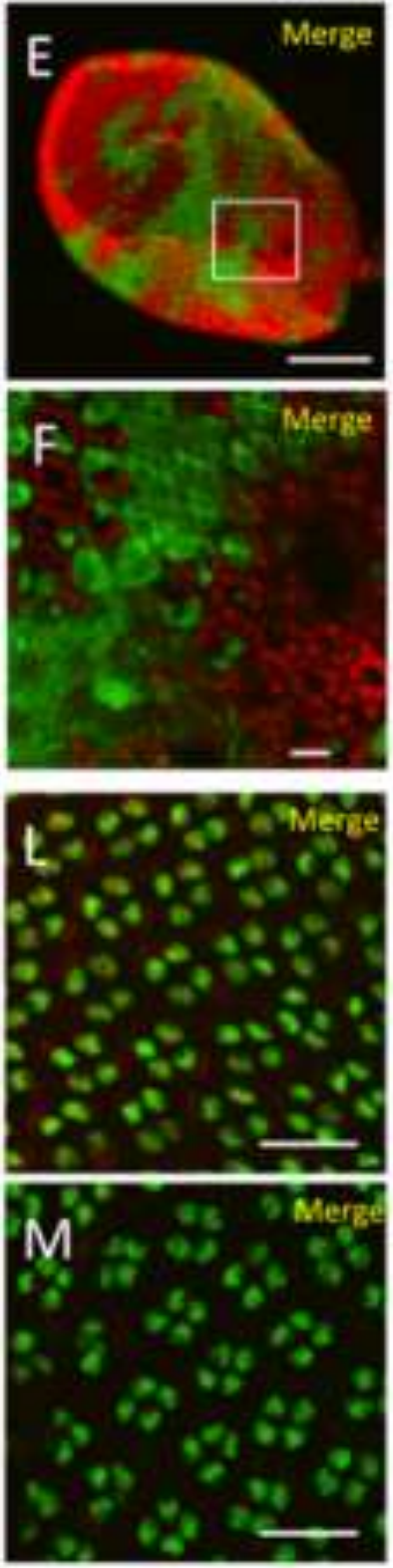
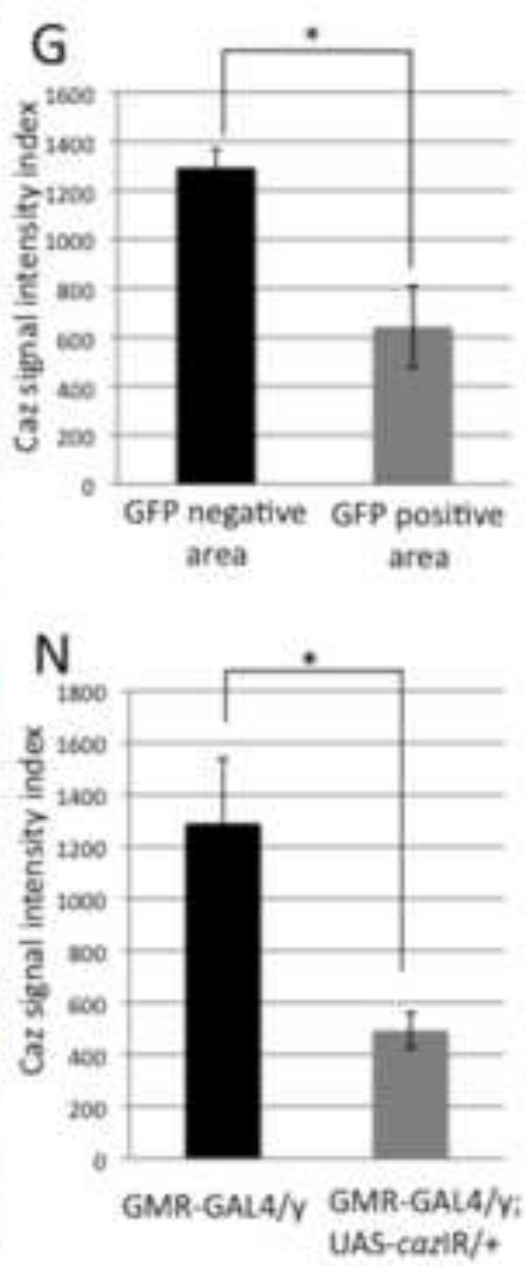

0

$42 \mathrm{kD}$ -

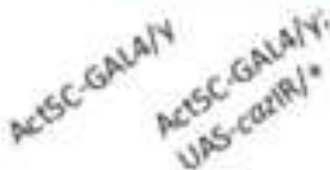



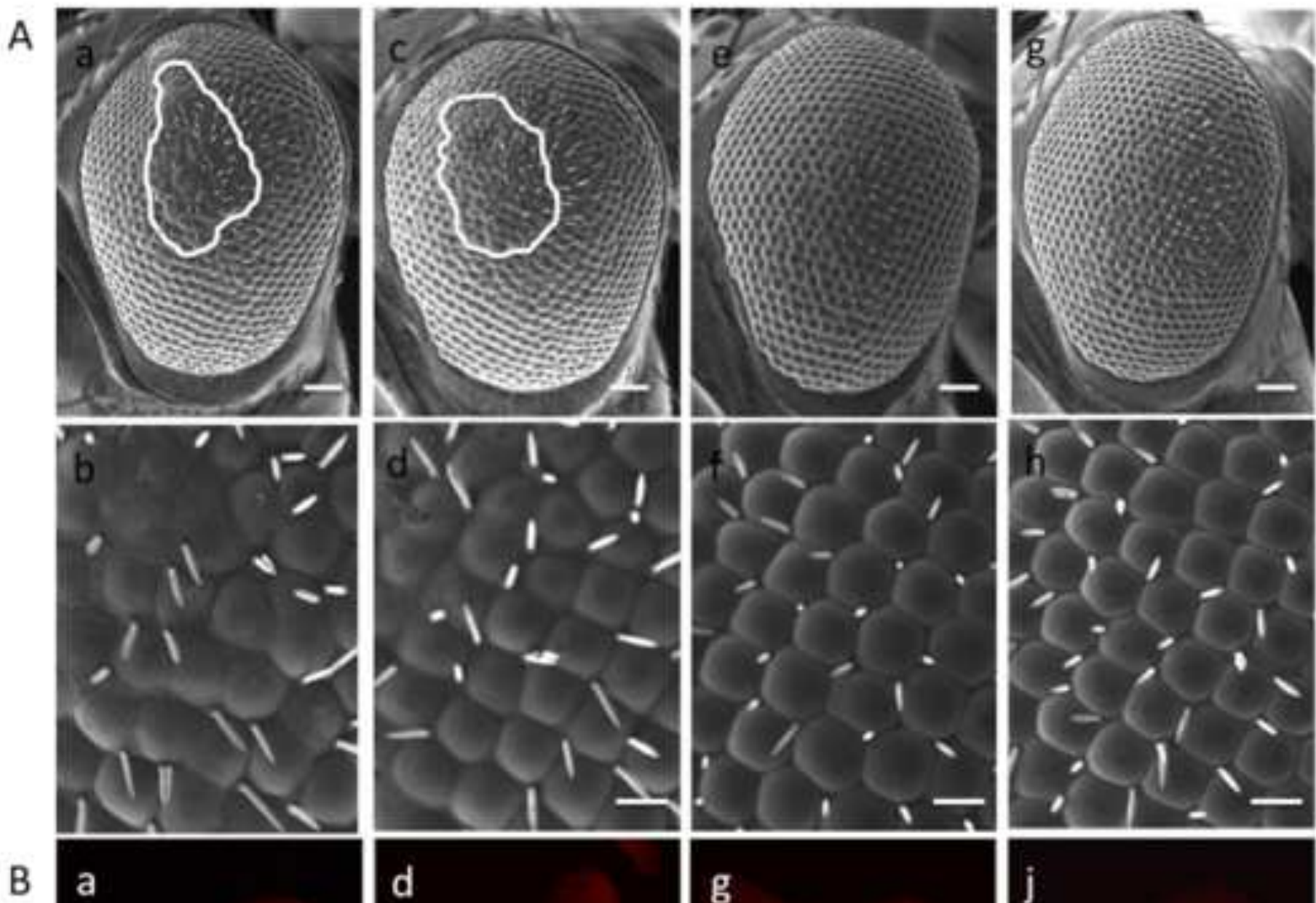

B

a
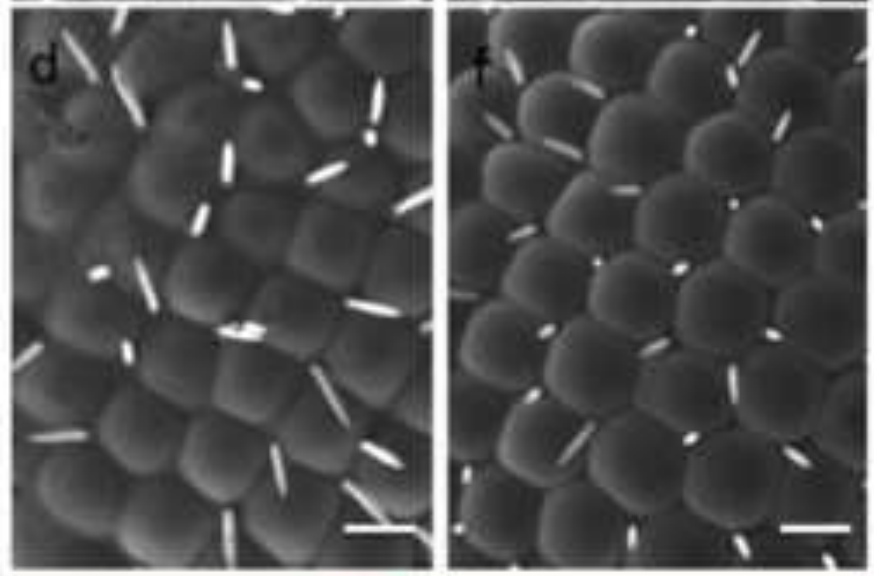

g
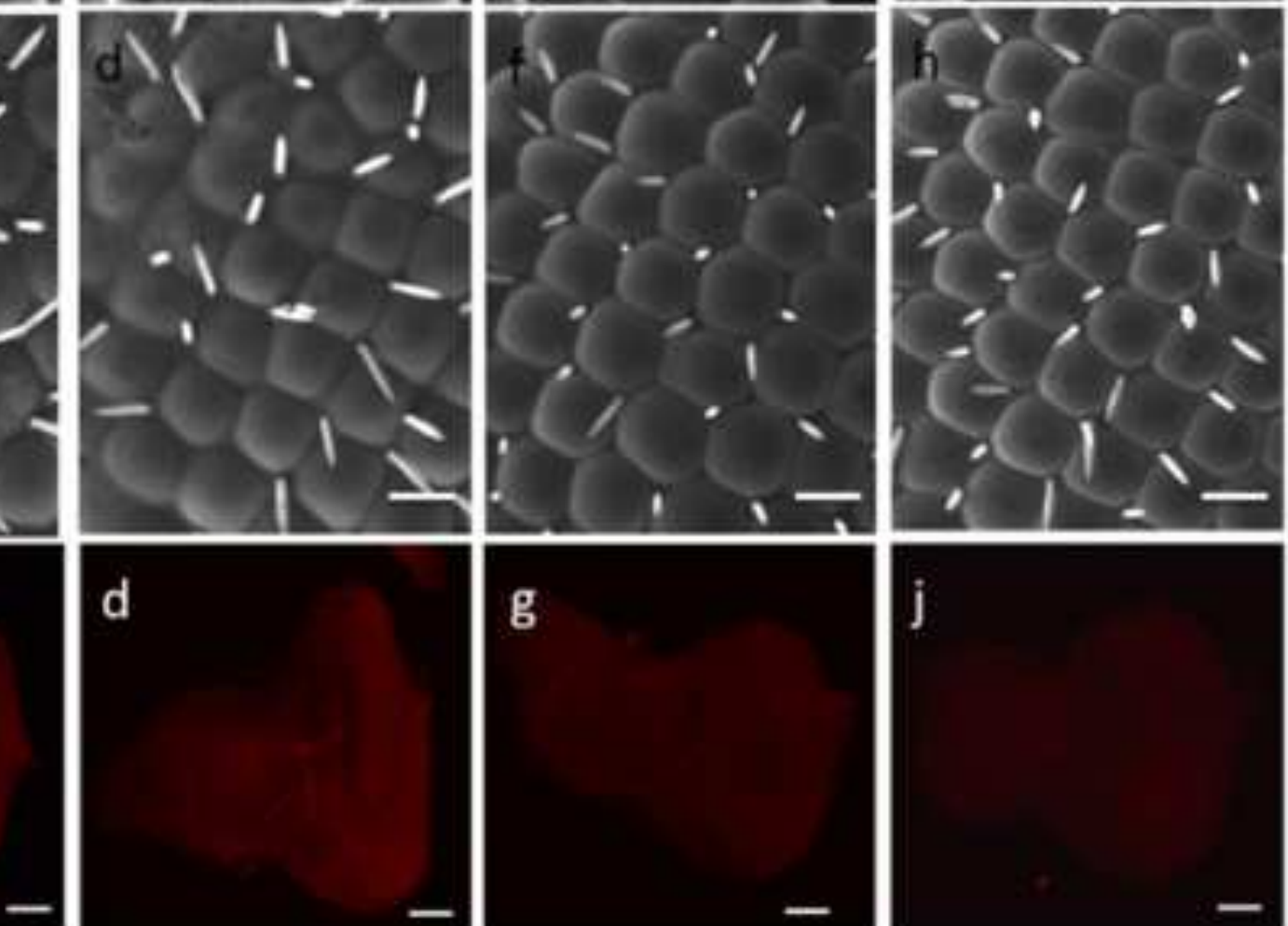

$-$

b
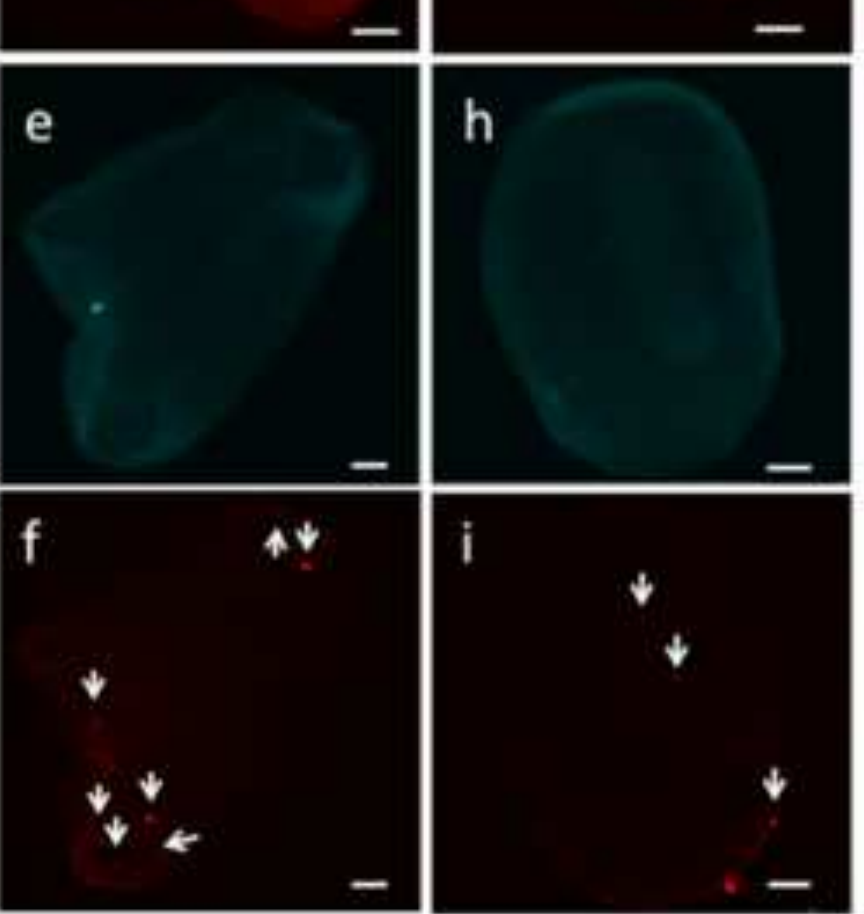

k
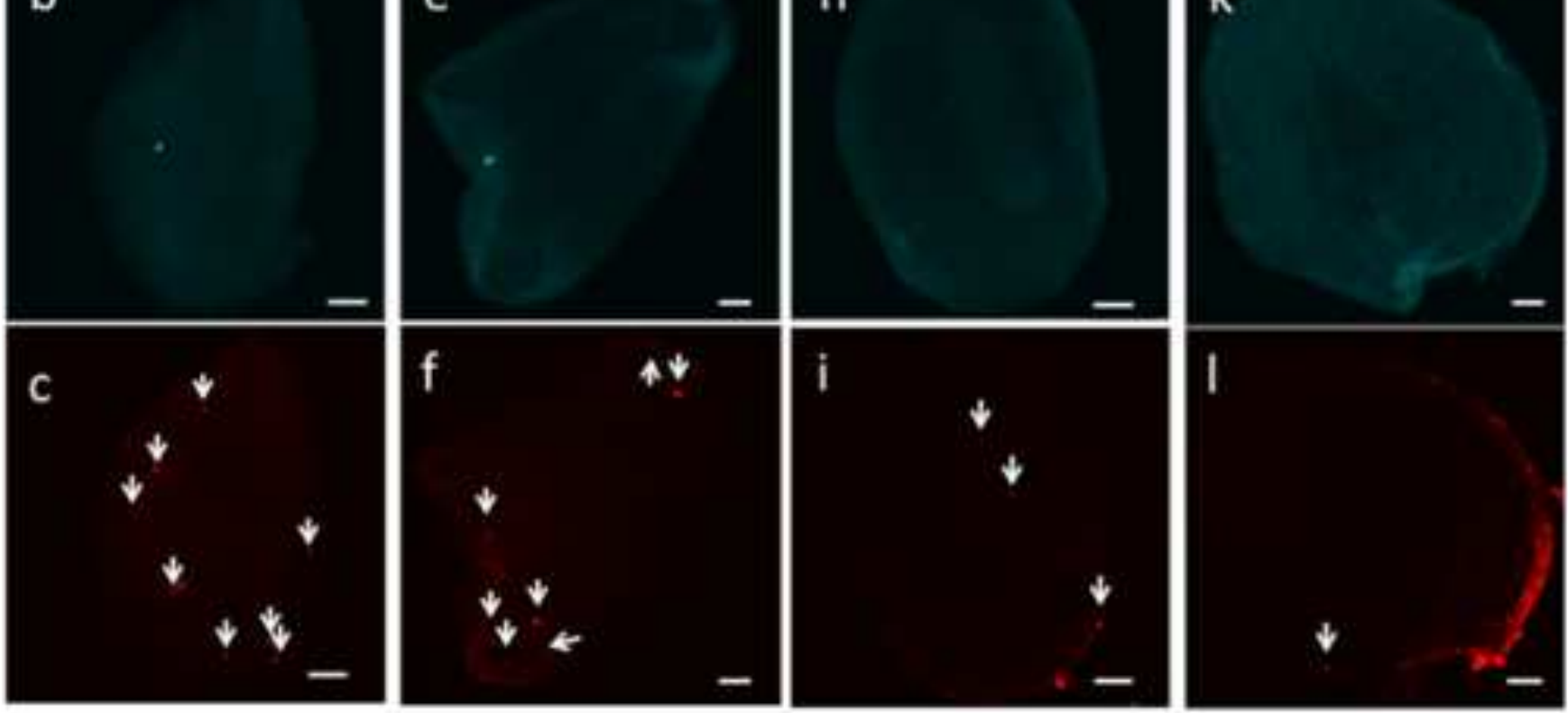

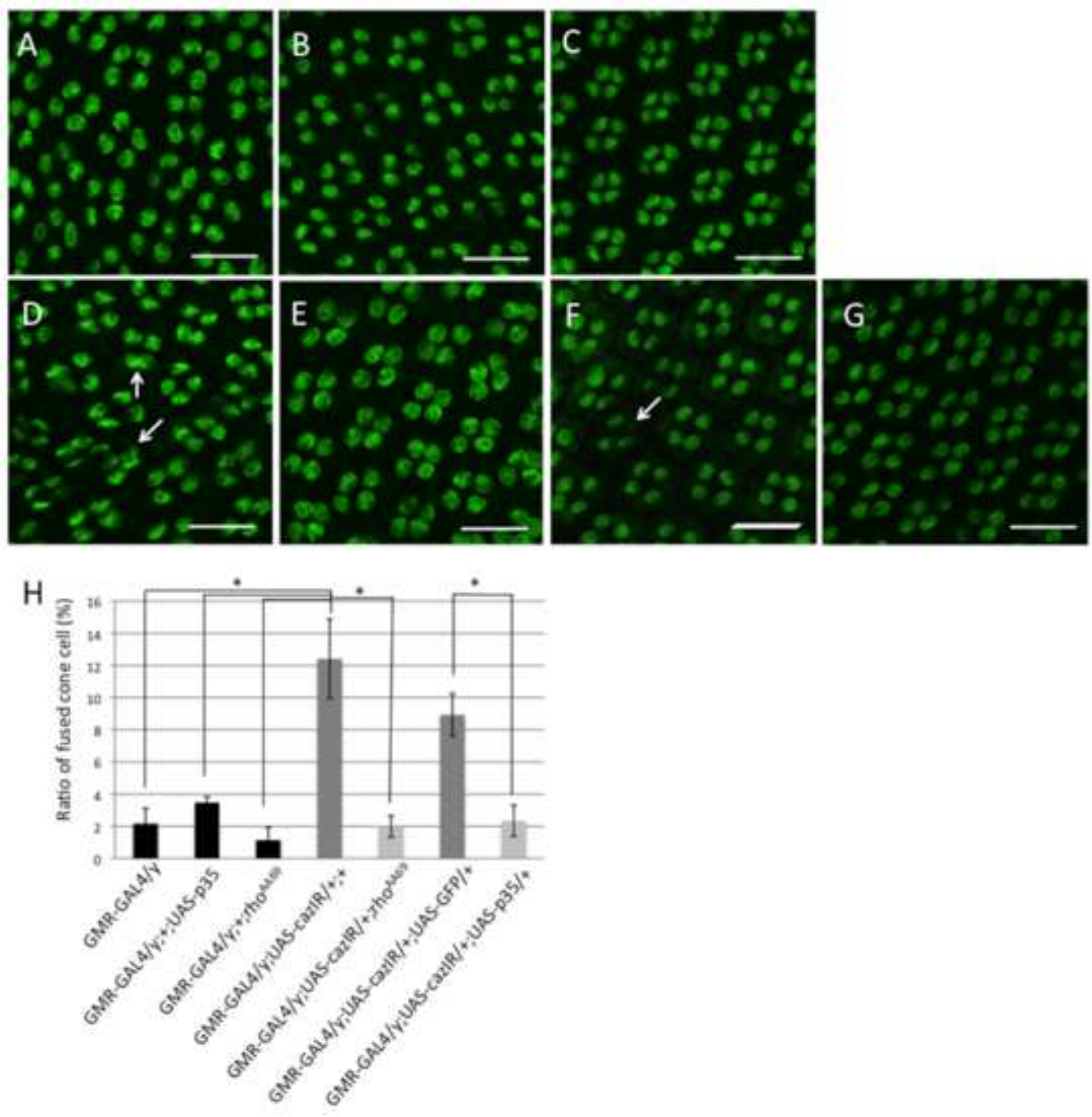

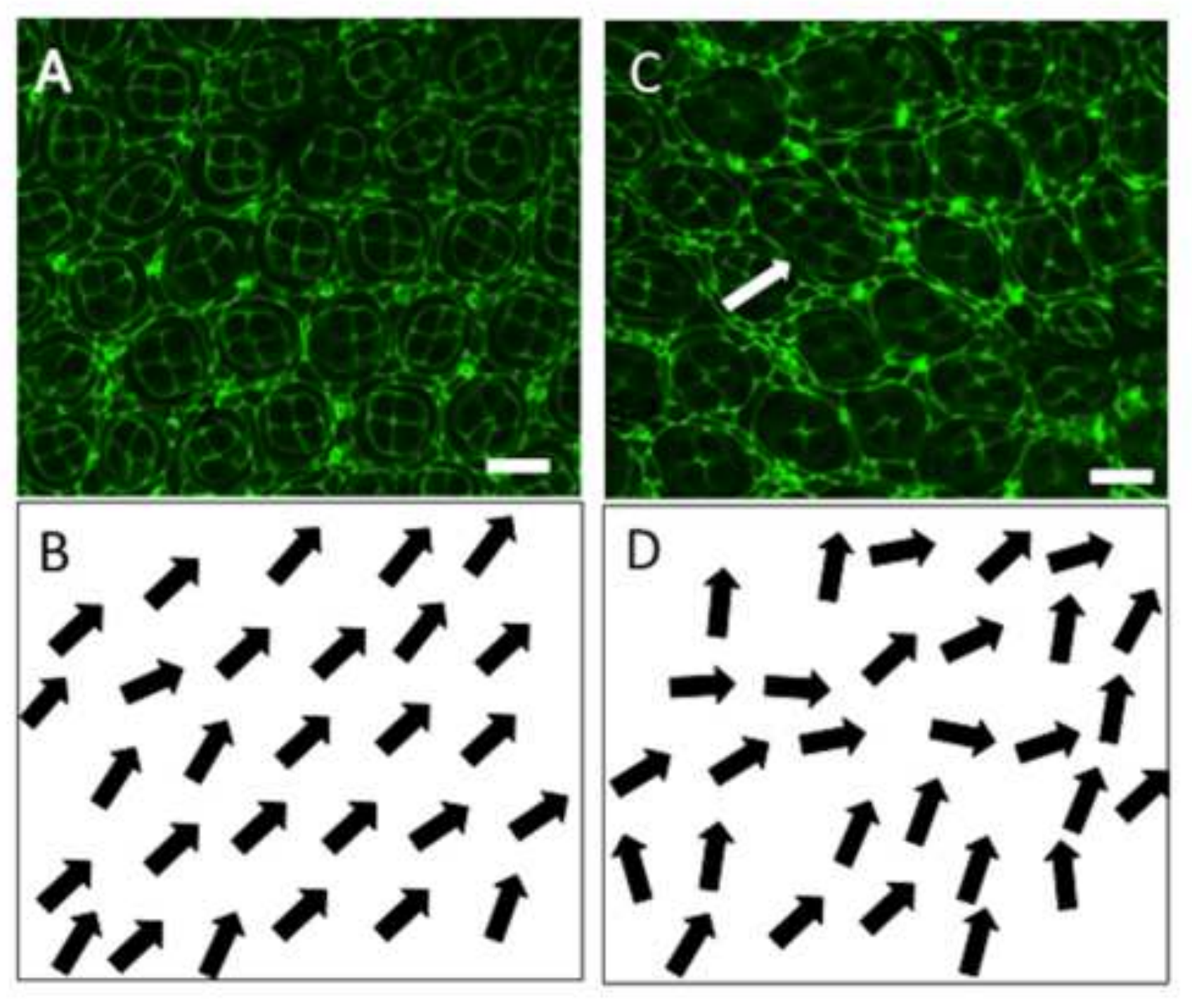

Figure4

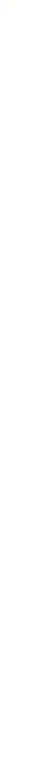

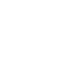

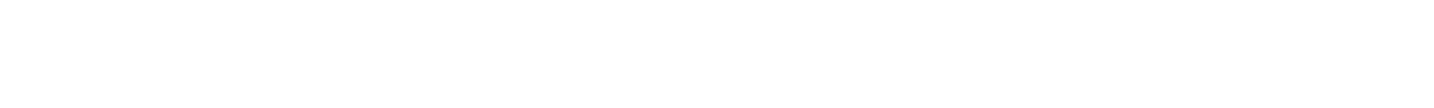


